\title{
The Influence of Various Intervention Types on Treatment Success Rates among Category II Tuberculosis Patients
}

\author{
Bandita SenGupta1, Carla J. Burrus ${ }^{2}$, Noor Tirmizi², Basab Rooj¹, Hilary S. Moshman', \\ Soumen Pandey ${ }^{1}$, Gundegmaa Jaamaa ${ }^{2}$, Khrist Roy ${ }^{2 *}$ \\ ${ }^{1}$ CARE India, Calcutta, India \\ ${ }^{2}$ CARE USA, Atlanta, GA, USA \\ Email: ${ }^{*}$ kroy@care.org
}

Received 20 April 2015; accepted 18 June 2015; published 23 June 2015

Copyright (C) 2015 by authors and Scientific Research Publishing Inc.

This work is licensed under the Creative Commons Attribution International License (CC BY). http://creativecommons.org/licenses/by/4.0/ c) (i) Open Access

\begin{abstract}
Background: Category II tuberculosis (TB) patients (i.e. re-treatment TB patients) are at an increased risk for defaulting on treatment compared to Category I TB patients. Therefore, extra steps need to be taken to help Category II TB patients follow through with their treatment. The goal of this study was to examine the effectiveness of three different types of interventions to help improve treatment success rates among Category II patients. Materials and Methods: Three different interventions that were implemented among Category II TB patients in the Bardhaman, Hugli, Malda and Murshidabad districts in West Bengal, India, were: 1) setting up group patient provider meetings (PPMs), 2) making home visits and reinforcing the message of full course of treatment, and 3) linking poor TB patients to social welfare schemes (SWSs) to incentivize them to complete treatment. Results: PPMs and SWSs improved treatment success rates among Category II patients. The treatment success rates for patients who received PPMs and patients who received SWSs were $94.2 \%$ and $\mathbf{9 0 . 7 \%}$, respectively, compared to the $74.5 \%$ treatment success rate of patients who received no intervention. The effectiveness of home visits, however, depended on the number of home visits the patient received. Conclusion: PPMs and SWSs improve treatment success among Category II TB patients and may easily be incorporated in Directly Observed Treatment, Short-Course programming as feasible ways. A conclusion regarding home visits, however, could not be drawn from this study.
\end{abstract}

\section{Keywords}

Treatment Default, Social Welfare Schemes, Group Patient Provider Meetings, Home Visits

\footnotetext{
${ }^{*}$ Corresponding author.
}

How to cite this paper: SenGupta, B., Burrus, C.J., Tirmizi, N., Rooj, B., Moshman, H.S., Pandey, M., Jaamaa, G. and Roy, K. (2015) The Influence of Various Intervention Types on Treatment Success Rates among Category II Tuberculosis Patients. Journal of Tuberculosis Research, 3, 43-49. http://dx.doi.org/10.4236/itr.2015.32006 


\section{Introduction}

Nearly 400,000 tuberculosis (TB) patients die in India annually even though there are cost-effective tools to cure the disease [1] [2]. In 1993, The Revised National Tuberculosis Control Programme (RNTCP) was implemented to help address this problem. The RNTCP is an application of the Directly Observed Treatment, Short-Course (DOTS) strategy, in which a health care worker observes and assists with the patient taking their medicine [1]. The DOTS treatment program, however, may intrude on the daily life obligations of patients. For instance, patients may have familial and work obligations that prevent them from traveling to a health center multiple times per week to receive and be observed taking their medicine. Therefore, many patients do not complete their entire course of treatment [3]. Thus, although RNTCP has improved treatment success rates (TSRs) among TB patients in India [4], many patients still tend to have poor treatment outcomes [5].

Category II patients (i.e., patients who have defaulted during a previous treatment, relapsed after treatment, or who have failed previous TB treatment [1]), in particular, are at increased risk for defaulting on their treatment [5]-[8], and for having multi-drug resistant strains [9]. In India, the Category II TSR is only 70\% [10] and in West Bengal it is even lower at $64 \%$ [11]. This is likely because the treatment for Category II patients often requires more effort on the part of the patient than Category I TB patient treatment. Specifically, Category II patient treatment may require a more intensive medication regimen and longer treatment duration. Additionally, these patients have already gone through an unsuccessful round of treatment at least once, and therefore, may be less motivated to complete treatment the second time around. Therefore, in addition to following the general RNTCP protocol, it may be beneficial to take extra steps with Category II TB patients in order to ensure that they complete their entire treatment course.

This study, funded by the United States Agency for International Development (USAID)/Child Survival and Health Grants Program (CSHGP), examined the effectiveness of three interventions on improving TSRs among Category II TB patients in West Bengal, India. These interventions were aimed at 1) linking Category II patients with each other and with their service providers to discuss their treatment and the issues they are having and 2) incentivizing patients to follow through with their entire treatment course. Specifically, first, a platform was created for group patient provider meetings (i.e., PPMs), which allowed for the interaction between groups of patients and a service provider. By having the group meetings, the provider (usually a block medical officer) was able to meet with the patients frequently to address their issues. Furthermore, the patients were able to provide one another psychological and social support while also sharing knowledge regarding their disease and its treatment. Second, home visits with the patient and their families were made during which the message of treatment compliance was reinforced, and the management of side-effects was discussed. Lastly, poor patients were linked with social welfare schemes (i.e., SWSs) provided by the Panchayati Raj Institutions (PRIs), the lowest level of self-governance in India. For example, the PRI could provide cash, food (e.g., Horlicks, rice), supplies (e.g., blankets), a combination of cash, food and/or supplies, or preferential employment through government relief programming and/or government employment schemes to incentivize patients to follow through with TB treatment.

\section{Materials and Methods}

\subsection{Study Implementation}

As part of the Initiative to Manage People Centered Alliances in Control of Tuberculosis (IMPACT) project, three different interventions (i.e. PPMs, SWSs, and home visits) were adopted to improve the TSRs among Category II patients. The implementation area for these three interventions included 22 Treatment Units (TUs) in West Bengal spread among four districts: Bardhaman, Hugli, Malda and Murshidabad. The selection of the TUs was based on two basic indicators: case detection rate and treatment success rate. Those TUs who performed the worst on these indicators were selected for the study. All 3310 Category II TB patients who registered during 2011 in these TUs formed the study population. However, only 2327 patients were included in the analysis because data was missing from the other 983 . Treatment success was defined as those patients who successfully completed treatment and are believed to no longer be infected with TB, and treatment default was defined as those patients who did not complete treatment.

A total of 54 block coordinators (BCs), one assigned to each block, were recruited by partner non-governmental organizations for implementing the project activities. In order to implement the three interventions, the 
BCs collected a list of all retreatment patients, and a home visit was initiated for them. The initial home visit was done by the Auxiliary Nurse Midwife (ANM). Subsequent home visits were made by IMPACT project staff within next fifteen days. During these visits an attempt was made to encourage patients to attend PPMs and to link them with existing SWSs through the PRI system. Thus, this study utilized a natural experiment in which an attempt was made to make at least one home visit to all patients within the study area, but the patients themselves chose whether they wanted to attend PPMs and the PRIs decided whether they wanted to offer SWSs to the TB patients within their local area. As such, the majority of patients received home visits $(\mathrm{N}=2237)$, fewer patients attended PPMs $(\mathrm{N}=1022)$, and even fewer received SWSs $(\mathrm{N}=830)$. Furthermore the majority of the patients received more than one intervention $(\mathrm{N}=2239)$. The breakdown of the number of patients who received each intervention is shown in Table 1.

\subsection{Data Collection}

Data was collected using the information on the TB patient register maintained by the BCs and the individual patient RNTCP TB cards, which are issued to all TB patients. These included information such as the patient's age, sex, caste, category, and treatment outcome. The register also included information regarding attendance in PPMs, the number of home visits made, and if the patient was linked to SWSs. If the patient consented, data collected also included information on poverty status. All personal identifiers were removed from the data.

\subsection{Analysis}

A binary logistic regression model was utilized to examine the influence of each of the interventions on TSRs compared to default rates. This type of analysis was used because we had a binary outcome variable (i.e. treatment success versus treatment default) and it allows for conclusions to be drawn regarding the effect of each of the three intervention types on the treatment outcome, while at the same time controlling for the influence of the other intervention types. This last point is important given that most patients in the study population received more than one intervention. Age, poverty level, and sex were included in the model as control variables. Finally, Tukey's HSD tests were conducted to assess the influence of the number of home visits on TSRs. All analyses that had p-values of .05 or below were considered significant.

\section{Results}

\subsection{Socio-Demographics}

The socio-demographics of the study population are presented in Table 2. As shown in the table, a majority of the patients included in this study were male (78.0\%), between the ages of 15 and 45 years old (62.8\%), of general caste (52.1\%), and living below the poverty line (54.2\%). Out of the patients included in this study, $43.9 \%$ attended PPMs, 35.7\% received SWSs, and 96.1\% received home visits.

\subsection{Intervention Effectiveness}

Our results indicated that two out of the three interventions significantly improved TSRs (see Figure 1 for the

Table 1. The number of patients who received each combination of interventions.

\begin{tabular}{cc}
\hline Interventions & $\mathrm{N}$ \\
\hline No Intervention & 55 \\
Only Patient Provider Meetings (PPMs) & 0 \\
Only Social Welfare Schemes (SWSs) & 33 \\
Only Home Visits & 862 \\
SWSs and PPMs & 2 \\
Home Visits and SWSs & 355 \\
Home Visits and PPM & 580 \\
Home Visits, PPM, and SWSs & 440 \\
\hline
\end{tabular}

Note: Total $\mathrm{N}=2327$. 
Table 2. Socio-demographic information by intervention.

\begin{tabular}{ccccccc}
\hline & \multicolumn{4}{c}{ Intervention } & \multicolumn{2}{c}{ Home Visits } \\
\cline { 2 - 7 } & \multicolumn{2}{c}{ Patient } & \multicolumn{2}{c}{ Provider } & Meetings & \multicolumn{2}{c}{ Social Welfare Schemes } & No & Yes & No \\
\cline { 2 - 7 } & Yes & No & Yes & 1497 & 2237 & 90 \\
Total N & 1022 & 1305 & 830 & 1743 & 71 \\
Male & 786 & 1028 & 637 & 1177 & 494 & 19 \\
Female & 236 & 277 & 193 & 320 & 33 & 2 \\
$<15$ years old & 12 & 23 & 18 & 17 & 1399 & 62 \\
15 - 45 years old & 636 & 825 & 528 & 933 & 805 & 26 \\
$46+$ years old & 374 & 457 & 284 & 547 & 1196 & 65 \\
BPL & 556 & 705 & 501 & 760 & 1010 & 25 \\
APL & 458 & 577 & 329 & 706 & 7170 & 42 \\
General & 547 & 665 & 478 & 734 & 725 & 26 \\
SC & 348 & 403 & 240 & 511 & 252 & 342 \\
ST & 127 & 237 & 112 & 252 & 22 \\
\hline
\end{tabular}

Note: Total N = 2327; BPL = Below Poverty Line; APL = Above Poverty Line; SC = Scheduled Caste; ST = Scheduled Tribe.

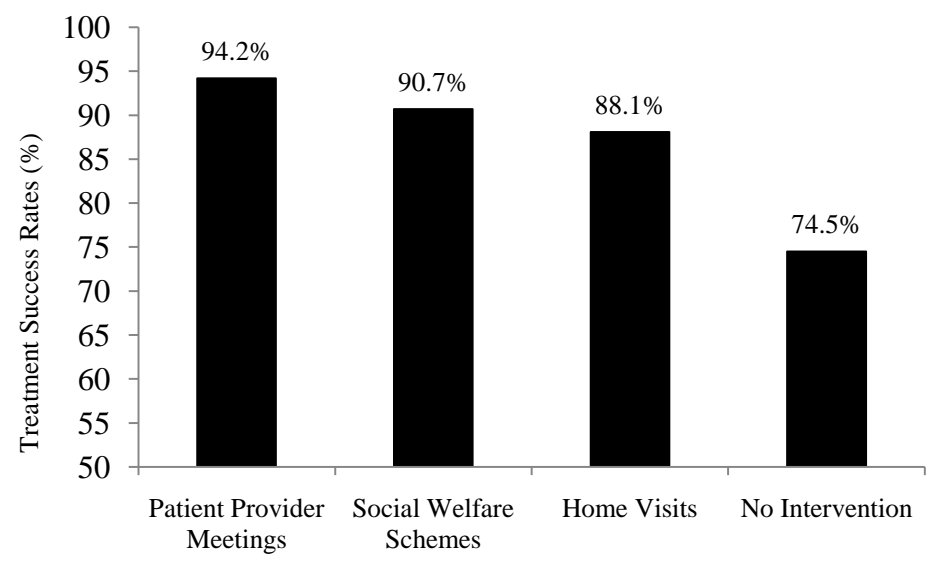

Figure 1. Treatment success rates for the different interventions. This graph shows the treatment success rates of the patients who received each type of intervention. Many patients received more than one intervention, and therefore, the percentages for each of the three interventions were not independent from each other. Instead, they reflected those patients who received that particular intervention but also may have received one of the other interventions as well.

TSRs for the patients who received each intervention). Specifically, Table 3 shows that TSRs improved with PPMs $(\operatorname{Exp}(B)=3.315, p<0.001)$, and SWSs incentives $(\operatorname{Exp}(B)=1.422, p<0.05)$, but not with home visits $(\operatorname{Exp}(B)=1.238, p>0.05)$.

\subsection{Number of Home Visits}

Although the results of the binary logistic regression indicated that home visits did not significantly influence TSRs, individual patients received different numbers of home visits (i.e., 90 patients received no home visits, 258 patients received one, 582 patients received two, and 1397 patients received three or more), and it was possible that a certain number of home visits were necessary to effectively improve TSRs. Indeed, Tukey's HSD tests showed that the number of home visits received had a significant effect on TSRs $(F(3 d f)=8.733, p<$ 0.001). Specifically, the TSRs from:

- Two home visits were significantly better than no home visits (Mean Difference $=-0.142, p<0.01$ ).

- Two home visits were significantly better than those from three or more home visits. (Mean Difference $=$ $0.071, p<0.001)$.

- No other comparisons (i.e., 0 home visits versus 1 home visit, 0 home visits versus 3 home visits, 1 home visit versus 2 home visits, and 1 home visit versus 3 home visits) were found to be significant. 
Table 3. Binary logistic regression analyses assessing the influence of the three interventions on treatment default $(0)$ and treatment success (1).

\begin{tabular}{|c|c|c|c|c|}
\hline \multirow{2}{*}{ Variable } & \multirow{2}{*}{ Comparison } & \multirow{2}{*}{$\operatorname{Exp}(B)$} & \multicolumn{2}{|c|}{$95 \%$ CI } \\
\hline & & & Lower & Upper \\
\hline \multirow{2}{*}{ Patient Provider Meetings } & Not Attended & & & \\
\hline & Attended & $3.315^{* * * *}$ & 2.437 & 4.511 \\
\hline \multirow{2}{*}{ Social Welfare Schemes } & No Received & & & \\
\hline & Received & $1.422^{*}$ & 1.067 & 1.895 \\
\hline \multirow{2}{*}{ Home Visits } & No & & & \\
\hline & Yes & 1.238 & 0.723 & 2.117 \\
\hline \multirow{2}{*}{ Gender } & Male & & & \\
\hline & Female & $1.661^{* *}$ & 1.165 & 2.368 \\
\hline \multirow{2}{*}{ Poverty Classification } & Above Poverty Line & & & \\
\hline & Below Poverty Line & $0.691^{*}$ & 0.514 & 0.928 \\
\hline \multirow{3}{*}{ Caste } & General & & & \\
\hline & Scheduled Caste & 0.900 & 0.661 & 1.225 \\
\hline & Scheduled Tribe & 0.881 & 0.604 & 1.286 \\
\hline \multirow{3}{*}{ Age } & 46 years old or above & & & \\
\hline & Less than 15 years old & 1.747 & 0.403 & 7.569 \\
\hline & 15 to 45 years old & 0.839 & 0.639 & 1.101 \\
\hline
\end{tabular}

Note: Nagelkerke $\mathrm{R}^{2}=8.8 \%$. Total $\mathrm{N}=2296,31$ had missing data for one or more of the variables included in the model.

\section{Discussion}

\subsection{Discussion of Results}

The results of study demonstrate the effectiveness of interventions on improving TSRs. These results are in line with the results of similar studies that show that interventions, such as the introduction of incentives, increase treatment success rates among TB patients [12]-[16]. For instance, Volimark et al. found that interventions such as reminder cards, monetary incentives, and health education all improved treatment compliance among TB patients [16]. Unlike these previous studies, however, this study examined the effectiveness of multiple types of interventions (including the novel intervention of PPMs) on the particularly high-risk population for treatment default-Category II TB patients. The results of the present study demonstrate the positive effects that PPMs and SWSs have on TSRs among Category II patients. Compared to those patients who did not receive any intervention (74.5\% treatment success), those patients who attended PPMs (94.2\% treatment success) and who received SWS incentive (90.7\% treatment success) had substantially improved TSRs.

SWSs provide incentives for following through with treatment. Therefore, the patients are more likely to complete the entire treatment course in order to continue to receive these incentives. PPMs are likely effective because the meetings allow patients to have a better understanding of the disease course and the importance of treatment. One of the primary reasons the patients default on treatment is that they stop seeing and feeling the symptoms of their TB over the course of their treatment, making it particularly important to talk to a health provider (block medical officer) who can help them understand the importance of completing their treatment [17]. It is possible, however, that PPMs increases TSRs due to participation bias such that these patients may have been more positively inclined to successfully complete treatment given that they are self-selected to attend the meetings.

Unlike SWSs and PPMs, results for home visits influencing TSRs are ambiguous. Although the TSRs of those patients who received home visits (88.1\% treatment success) were higher than the TSRs of those patients who did not receive any intervention, the binary linear regression revealed that this difference was not significant. However, further analysis (Tukey's HSD test) revealed that the number of home visits the patient received influenced TSRs such that TSRs improved the most when two home visits were conducted. It is unclear, however, 
why this would be the case. One potential reason that only two home visits were effective at improving treatment success rates could have been because one home visit was not enough, but those patients who received three or more home visits may have been particularly "high risk" patients for treatment default to begin with (thus the need for several home visits). In such a case, home visits alone might not be enough to motivate those patients who are at the highest risk of default to follow through with treatment. That being said, firm conclusions regarding the effectiveness of home visits in improving TSRs cannot be drawn based on this study. Ideally, future studies would be conducted that systematically assign patients to a given number of home visits in order to more accurately assess the influence that the number of home visits has on treatment success rates.

\subsection{Limitations}

Although this study provides some insight into the effectiveness of various interventions on treatment success rates among Category II TB patients, it does have several limitations. Specifically, given that this study took advantage of a natural experiment, and patients were not assigned to various intervention types or combinations of interventions, the relative influence of each intervention type could not be compared because the number of patients who received each intervention combination varied greatly from 0 - 862 patients. Furthermore, given that participation in the interventions was self-selected, participation bias could have influenced the treatment outcomes associated with each intervention rather than the intervention itself being the sole influence. Finally, this study only took place within four districts in West Bengal, India. Thus, the results may not be generalizable to Category II TB patients outside of these districts. Future studies should systematically assign patients to each intervention type so that direct comparisons can be made among the relative effectiveness of each intervention types and participation bias can be eliminated. Additionally, similar studies should be conducted in other geographical areas in which Category II patient default rates are high in order to examine the generalizability of these results.

\section{Conclusion}

Based on the results of this study, we believe that PPMs and SWSs may easily be incorporated in DOTS programming and have the potential to decrease treatment default rates and consequently improve TSRs among Category II TB patients. Furthermore, the same interventions may also improve TSRs among other high risk patients for treatment default including alcoholics, or those patients without a convenient treatment facility [5] [9] [18] [19].

\section{Acknowledgements}

The authors would like to acknowledge RNTCP, CARE, German Literacy, TB Relief Associations (GLRA), Southern Health Improvement Samity (SHIS), and USAID/CSHGP for their support.

\section{References}

[1] Agarwal, S.P. and Chauhan, L.S. (2005) Tuberculosis Control in India. Directorate General of Health Services, Ministry of Health and Family Welfare, New Delhi.

[2] Dye, C., Scheele, S., Dolin, P., Pathania, V. and Raviglione, M.C. (1999) The WHO Global Surveillance and Monitoring Project. Global Burden of Tuberculosis: Estimated Incidence, Prevalence, and Mortality by Country. JAMA, 282, 677-686. http://dx.doi.org/10.1001/jama.282.7.677

[3] Jaiswal, A., Singh, V., Ogden, J.A., Porter, J.D.H., Sharma, P.P., Sarin, R., et al. (2003) Adherence to Tuberculosis Treatment: Lessons from the Urban Setting of Delhi, India. Tropical Medicine \& International Health, 8, 625-633. http://dx.doi.org/10.1046/j.1365-3156.2003.01061.x

[4] Khatri, G.R. and Frieden, T.R. (2000) The Status and Prospects of Tuberculosis Control in India. International Journal of Tuberculosis and Lung Disease, 4,193-200.

[5] Chadha, S.L. and Bhagi, R.P. (2000) Treatment Outcome in Tuberculosis Patients Places under Directly Observed Treatment Short Course (DOTS)—A Cohort Study. Indian Journal of Tuberculosis, 47, 155-158.

[6] Jaggarajamma, K., Sudha, G., Chandrasekaran, V., Nirupa, C., Thomas, A., Santha, T., et al. (2007) Reasons for NonCompliance among Patients Treated under Revised National Tuberculosis Control Program (RNTCP), Tiruvallur District, South India. Indian Journal of Tuberculosis, 54, 130-135.

[7] Mittal, C. and Gupta, S.C. (2011) Noncompliance to DOTS: How It Can Be Decreased. Indian Journal of Community 
Medicine, 36, 27-30. http://dx.doi.org/10.4103/0970-0218.80789

[8] Dooley, K.E., Lahlou, O., Ghali, I., Knudsen, J., Elmessaoudi, M.D., Cherkaoui, I. and Aouad, R.E. (2011) Risk Factors for Tuberculosis Treatment Failure, Default, or Relapse and Outcomes of Retreatment in Morocco. BMC Public Health, 11, 140-147. http://dx.doi.org/10.1186/1471-2458-11-140

[9] Sharma, S.K., Kumar, S.K., Saha, P.K., George, N., Arora, S.K., Gupta, D., et al. (2011) Prevalence of Multidrug-Resistant Tuberculosis among Category II Pulmonary Tuberculosis Patients. Indian Journal of Medical Research, 133, 312-315.

[10] (2014) TB India 2014: Revised National TB Control Program, Annual Status Report. Central TB Division, Directorate General of Health Services, Ministry of Health \& Family Welfare, New Delhi, India.

[11] Government of West Bengal (2013) RNTCP Performance Report for West Bengal $4^{\text {th }}$ Quarter 2013.

[12] Giuffrida, A. and Torgerson, D.J. (1997) Should We Pay the Patient? Review of Financial Incentives to Enhance Patient Compliance. BMJ, 315, 703-707. http://dx.doi.org/10.1136/bmj.315.7110.703

[13] Buchanan, R.J. (1997) Compliance with Tuberculosis Drug Regimens: Incentives and Enablers Offered by Public Health Departments. American Journal of Public Health, 87, 2014-2017. http://dx.doi.org/10.2105/AJPH.87.12.2014

[14] Meichenbaum, D. (1987) TD. Facilitating Treatment Adherence: A Practitioner's Guidebook. Plenum, New York.

[15] Pozsik, C.J. (1989) Using Incentives and Enablers in the Tuberculosis Control Program. In: Pozsik, C., Ed., Tuberculosis Control: Enablers and Incentives, American Lung Association of South Carolina, Columbia.

[16] Volmink, J. and Garner, P. (1997) Systematic Review of Randomised Controlled Trials of Strategies to Promote Adherence to Tuberculosis Treatment. BMJ, 315, 1403-1406. http://dx.doi.org/10.1136/bmj.315.7120.1403

[17] Santha, T., Garg, R., Frieden, T.R., Chandrasekaran, V., Subramani, R., Gopi, P.G., et al. (2002) Risk Factors Associated with Default, Failure and Death among Tuberculosis Patients Treated in a DOTS Programme in Tiruvallur District, South India, 2000. International Journal of Tuberculosis and Lung Disease, 6, 780-788.

[18] Joseph, P., Chandrasekaran, V., Thomas, A., Gopi, P.G., Rajeswari, R., Balasubramanian, R., et al. (2006) Influence of Drug Susceptibility on Treatment Outcome and Susceptibility Profile of "Failures" to Category II Regimen. Indian Journal of Tuberculosis, 53, 141-148.

[19] Misha, P., Hansen, E.H., Sabroe, S. and Kafle, K.K. (2005) Socio-Economic Status and Adherence to Tuberculosis Treatment: A Case-Control Study in a District of Nepal. International Journal of Tuberculosis and Lung Disease, 9, 1134-1139. 